\title{
Predictors of Change in CD4 Count among Adult HIVIAIDS Patients on Anti-Retroviral Treatment in West Hararghe Zone, Ethiopia; Retrospective Longitudinal Study
}

\author{
Adisu Birhanu Weldesenbet ${ }^{1,}$, , Biruk Shalmeno Tusa ${ }^{1}$, Sewnet Adem Kebede ${ }^{2}$, \\ Getachew Asfaw Dagne ${ }^{3}$ \\ ${ }^{1}$ Department of Epidemiology and Biostatistics, Collage of Health and Medical Sciences, Haramaya University, Haramaya, Ethiopia \\ ${ }^{2}$ Department of Epidemiology and Biostatistics, Institute of Public Health, College of Medicine and Health Sciences, University of Gondar, \\ Gondar, Ethiopia \\ ${ }^{3}$ College of Public Health, University of South Florida, Tampa, United State of America
}

\section{Email address:}

adisuebi2009@gmail.com (A. B. Weldesenbet), birukshalmeno27@gmail.com (B. S. Tusa), sewnetme1@gmail.com (S. A. Kebede), gdagne@ health.usf.edu (G. A. Dagne)

*Corresponding author

\section{To cite this article:}

Adisu Birhanu Weldesenbet, Biruk Shalmeno Tusa, Sewnet Adem Kebede, Getachew Asfaw Dagne. Predictors of Change in CD4 Count among Adult HIV/AIDS Patients on Anti-Retroviral Treatment in West Hararghe Zone, Ethiopia; Retrospective Longitudinal Study. International Journal of HIV/AIDS Prevention, Education and Behavioural Science. Vol. 6, No. 2, 2020, pp. 32-37. doi: $10.11648 /$ j.ijhpebs.20200602.11

Received: June 3, 2020; Accepted: June 17, 2020; Published: July 4, 2020

\begin{abstract}
Background: As one of the countries in the Sub-Saharan African Region, Ethiopia also happens to bear a higher burden of HIV infection. For HIV-infected patients, the level of CD4 count remains an important test with regard to diagnostic decision-making. There is limited information on predictors of longitudinal change in CD4 count over time that examine immunologic response of patients during the course of treatment in Ethiopia. Therefore, this study aimed to examine predictors of change in CD4 count among adult HIV infected patients on antiretroviral treatment in west Hararghe zone, Ethiopia. Methods: An institutional based retrospective cohort study was conducted among 405 adult HIV/AIDS patients on Anti-Retro Viral Therapy (ART) from September 2013 to January 2019. Data was entered into Epi info 7 and analyzed in R software. Generalized mixed effect model was applied to identify predictors of longitudinal change in CD4 count. Results: In multivariable analysis time since start of ART (beta $=0.306,95 \% \mathrm{CI}, 0.286: 0.326)$, primary level educational status $($ beta $=0.048$, 95\%CI, 0.004: 0.092), tertiary educational status (beta $=0.094,95 \% \mathrm{CI}, 0.007: 0.182)$, WHO RVI stage II (beta $=-0.108,95 \% \mathrm{CI}$, $-0.156:-0.061$ ), bedridden functional status (beta $=-0.175,95 \% \mathrm{CI},-0.309:-0.039)$, poor baseline adherence (beta $=-0.145,95 \% \mathrm{CI}$, $-0.214:-0.076$ ) and baseline weight (beta $=-0.004,95 \% \mathrm{CI},-0.006,-0.002$ ) were significant predictors of longitudinal CD4 change. Conclusion: In this study time since start of ART, primary and tertiary educational status contributed positively to the change in CD4 count whereas bedridden functional status, poor adherence, WHO RVI stage II and baseline weight are negatively associated with longitudinal change in CD4 count. Close monitoring for bedridden patients and patients with poor baseline adherence is needed especially during the initiation of ART for immunological response.
\end{abstract}

Keywords: HIV/AIDS, CD4 Count, Generalized Linear Mixed Model, ART

\section{Introduction}

Globally, 37.9 million people are living with HIV until the end of 2018 of which 36.2 million are adults [1]. HIV/AIDS is a major public health problem in Sub-Saharan Africa since the region bears the highest burden of HIV infection. Ethiopia, as one of these countries has been affected by the epidemic with overall prevalence of $1.5 \%$ and prevalence of $1 \%$ among adults. In 2018, 690000 individuals were living with HIV, of which 23,000 were newly infected with HIV and 11,000 died from an AIDS-related illness in Ethiopia [2, 3].

Patients with retro viral infection (RVI) presented with 
advanced HIV disease need to be identified for prophylaxis against opportunistic infections and intensive monitoring for immune recovery. In addition when viral suppression and ARV drug resistance happens CD4 may be depleted and need CD4 count guided management [4]. CD4 count remains an important test with regard to diagnostic decision-making particularly for patients with advanced HIV disease. Monitoring of CD4+ cell counts over time provides a surrogate measure of HIV disease progression [5, 6].

Because HIV disease compromise the immune system by affecting the CD4 cell counts it can be employed to make appropriate decisions for the initiation of ART, identifying degree of immunosuppression and proper management of the progression of the infection [7]. It is an essential component of HIV treatment and care programmes and help healthcare providers by guiding key clinical decisions ranging from when to start (ART) to whether or not to screen for or provide prophylaxis against opportunistic infections [8,9].

Despite the above facts there is limited information on longitudinal change in CD4 count over time and its determinants that examine immunologic response of patients during the course of treatment in Ethiopia. Factors associated with CD4 change in HIV patients are multiple and complex and there is disagreement between studies on factors related to CD4 change like sex and age. Moreover previous studies failed to assess the effect of TB co-infection, weight and treatment regimen.

Therefore, this study aimed to examine predictors of change in CD4 count among adult HIV infected patients on antiretroviral treatment in west Hararghe zone, Ethiopia. The finding would be helpful for health professionals and patients to facilitate proper management by monitoring effectiveness of ART. In addition it would provide support for possible therapeutic interventions and, could result in a better quality of life and survival for patients.

\section{Methods}

\subsection{Study Design and Settings}

An institutional based retrospective cohort study was conducted among adult HIV/AIDS patients on ART from September 2013 to January 2019 at public hospitals in West Hararghe zone of Oromia region, Ethiopia. Among five hospitals in the zone the study was conducted in Chiro Zonal hospital and Gelemso General Hospital because of availability of the ART data. The two hospitals render services to around 2,300,000 population of the zone. At time of the study a total of 413 from Chiro Zonal Hospital and 221 patients from Gelemso General Hospitals are on ART.

\subsection{Study Population}

The study included all adult HIV/AIDS patients who started ART between 2013 and 2019 and registered in the ART registry log book from Chiro and Gelemso Hospitals. Patients who had at least two CD4 count measurements were included in the study. From HIV positive adults who started ART in the two selected hospital from September 2013 to January 2019,
405 patients who meet inclusion criteria were selected.

\subsection{Data Collection Procedures}

The data for this study was collected using a data extraction checklist. A baseline and follow up CD4 cell count data was identified and collected from the registration logbook of HAART attendants. Similarly, socio demographic variables, visiting times and clinical data were collected from the registration documents of patients. The data were collected by health professional after they had been given adequate orientation.

\subsection{Measurement of Study Variables}

Dependent variable for this study was CD4 cell count change. CD4 cell count change is defined as the difference between CD4 cells $/ \mathrm{mm}^{3}$ in the current visiting time and CD4 cells $/ \mathrm{mm}^{3}$ in visiting times immediately prior to the current response. Socio demographic variables (age, gender, marital status, educational status, occupational status, place of residence), baseline clinical variables (WHO RVI stage, base line weight, initial ART regimen, TB co-infection, opportunistic infection prophylaxis, baseline functional status and baseline adherence) were independent variables.

\subsection{Data Quality Assurance}

By conducting preliminary review adequacy of checklist was evaluated and variables on which data are unavailable were excluded from checklist. Trained health professionals were assigned as data collectors. Additionally, to ensure data quality filled checklist was checked for consistency and completeness. Strict supervision was applied by supervisors during data collection.

\subsection{Data Processing and Analysis}

The data entry was done using Epi Info 7 and then exported to $\mathrm{R}$ statistical software 3.6.2 for further analysis. Descriptive statistics such as mean, median, standard deviations (SDs) and tables were used to investigate the characteristics of the study participant. Generalized linear mixed effect model (GLMM) with log link function was used to identify predictors of changes in CD4 cell count. Both the random intercept and slope-intercept models were examined to see the model fit. Model comparison was done using likelihood ratio test.

We conducted bi-variable analyses to examine the relationship between each independent variable and longitudinal CD4 count measurement. Variables that were statistically significant at $\mathrm{p}$ values of 0.2 in bi-variable analyses were included in the multivariable analysis. Variables that were not statistically significant were excluded from the model with the exception of variables shown in previous studies to influence the outcome.

For multivariable analysis we used generalized linear mixed effect model with log link function predictors significantly associated with longitudinal CD4 change. Regression coefficients (beta) of the final model and their $95 \%$ confidence intervals were used as measures of association between the 
predictors and outcome variable. A p value of less or equal to 0.05 was considered statistically significant.

\section{Results}

A total of 405 patients on ART who meet inclusion criteria were included in the study of which $61.23 \%$ were from Chiro Zonal Hospital and $38.77 \%$ were from Gelemso General Hospitals. The median duration on ART and age of study participant was 39 months and 34 years (IQR, 27.0-40.0) respectively. Majority of the study subjects were females $(65.4 \%), 60 \%$ were from urban area and $55.1 \%$ were married. Mean baseline weight $( \pm \mathrm{SD})$ was $53.10(10.48) \mathrm{Kg}$, and $337(83.2 \%)$ participants had working baseline functional status, $330(81.5 \%)$ had TB co-infection and $19(4.7 \%)$ were in WHO stage IV (Table 1).

Table 1. Baseline characteristics of RVI patients on ART in West Hararghe zone, Ethiopia, 2013 to 2019.

\begin{tabular}{|c|c|c|c|}
\hline Variables & Category & $\begin{array}{l}\text { Frequency } \\
(n=405)\end{array}$ & $\begin{array}{l}\text { Percentage } \\
(\%)\end{array}$ \\
\hline \multirow{2}{*}{ Sex } & Male & 140 & 34.6 \\
\hline & Female & 265 & 65.4 \\
\hline \multirow{5}{*}{ Marital status } & Single & 46 & 11.4 \\
\hline & Married & 223 & 55.1 \\
\hline & Divorced & 57 & 14.1 \\
\hline & Widowed & 48 & 11.9 \\
\hline & Separated & 31 & 7.7 \\
\hline \multirow{7}{*}{$\begin{array}{l}\text { Occupational } \\
\text { status }\end{array}$} & Government employed & 34 & 8.4 \\
\hline & $\begin{array}{l}\text { Non-government } \\
\text { employed }\end{array}$ & 96 & 23.7 \\
\hline & Farmer & 87 & 21.5 \\
\hline & Student & 26 & 6.4 \\
\hline & Housewife & 94 & 23.2 \\
\hline & Merchant & 53 & 13.1 \\
\hline & Other & 15 & 3.7 \\
\hline \multirow{3}{*}{$\begin{array}{l}\text { Place of } \\
\text { residence }\end{array}$} & Urban & 243 & 60.0 \\
\hline & Rural & 162 & 40.0 \\
\hline & Uneducated & 129 & 31.9 \\
\hline \multirow{4}{*}{$\begin{array}{l}\text { Educational } \\
\text { status }\end{array}$} & Primary & 154 & 38.0 \\
\hline & Secondary & 77 & 19.0 \\
\hline & Tertiary & 43 & 10.6 \\
\hline & Diploma and above & 2 & 0.5 \\
\hline \multirow{4}{*}{ RVI stage } & Stage I & 207 & 51.1 \\
\hline & Stage II & 58 & 14.3 \\
\hline & Stage III & 121 & 29.9 \\
\hline & Stage IV & 19 & 4.7 \\
\hline \multirow{3}{*}{ Functional } & Working & 337 & 83.2 \\
\hline & Ambulatory & 55 & 13.6 \\
\hline & Bedridden & 13 & 3.2 \\
\hline \multirow{3}{*}{ Adherence } & Good & 353 & 87.2 \\
\hline & Fair & 18 & 4.4 \\
\hline & Poor & 34 & 8.4 \\
\hline \multirow{2}{*}{ TB co-infection } & Yes & 75 & 18.5 \\
\hline & No & 330 & 81.5 \\
\hline \multirow{2}{*}{ OIP } & Yes & 361 & 89.1 \\
\hline & No & 44 & 10.9 \\
\hline \multirow{4}{*}{ ART regimen } & AZT, 3TC, NVP & 23 & 5.7 \\
\hline & AZT, 3TC, EFV & 19 & 4.7 \\
\hline & TDF, 3TC, NVP & 17 & 4.2 \\
\hline & TDF, 3TC, EFV & 346 & 85.4 \\
\hline
\end{tabular}

Other=(Daily laborer, Driver).

\subsection{Exploring Changes in CD4 Cell Count}

A minimum of two and a maximum of four CD4 count measurements were taken for patients on ART during study period. Figure $1 \&$ Figure 2 presents individual profile plots and mean profile plot indicating that there is variability of CD4 count within and between patients. At baseline, patients had different starting points for CD4 count, suggesting a random intercept model. Looking at Figure 1, trajectories of CD4 over time show considerable heterogeneity which suggests to consider a mixed model with random slopes.

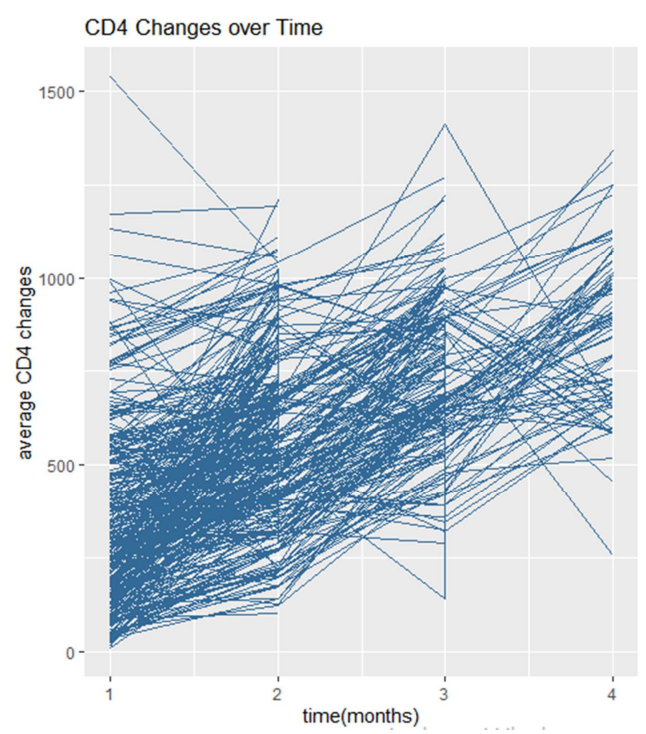

Figure 1. Individual profile plot for longitudinal CD4 trajectory among RVI patients on ART at public hospitals in west Hararghe zone, Ethiopia, 2013 to 2019.

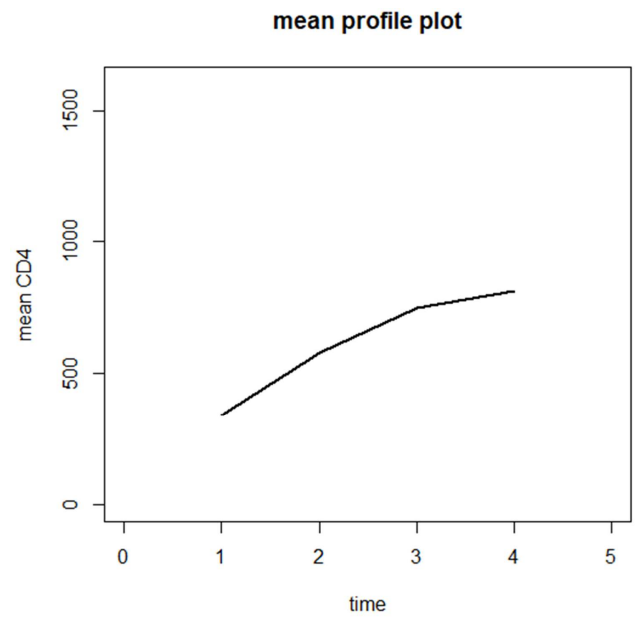

Figure 2. Mean profile plot for longitudinal CD4 trajectory among RVI patients on ART at public hospitals in west Hararghe zone, Ethiopia, 2013 to 2019

\subsection{Model Comparison}

In order to choose between random intercept only mixed model and random intercept and slope mixed model, a likelihood ratio test was carried out (Table 2). The result shows that random intercept and slope mixed model is a 
better model to fir the data.

Table 2. Shows model comparison for longitudinal models using Likelihood ratio test.

\begin{tabular}{lllll}
\hline Model & AIC & Log-Likelihood & Chi-square & P-value \\
\hline Random intercept model & 128475 & -64213 & & \\
Random intercept and slope model & 79551 & -39748 & 48928 & $<0.001$ \\
\hline
\end{tabular}

Predictors of Longitudinal CD4 cell count change

We fitted generalized linear mixed effect model with log link function to identify predictors of longitudinal CD4 count by adjusting for the effect of the covariates which were significantly associated with longitudinal change in CD4 in bi-variable analysis at $\mathrm{p}$ value of 0.2 (educational status, occupational status, place of residence, sex, baseline weight, WHO RVI stage, baseline adherence, duration on ART, TB co-infection, and functional status).
Table 3 shows in multivariable analysis time since start of ART (beta $=0.306,95 \% \mathrm{CI}, 0.286: 0.326$ ), primary level educational status (beta $=0.048,95 \% \mathrm{CI}, 0.004: 0.092$ ), tertiary educational status (beta $=0.094,95 \% \mathrm{CI}, 0.007: 0.182$ ), WHO stage II (beta $=-0.108,95 \% \mathrm{CI},-0.156:-0.061$ ), bedridden functional status (beta $=-0.175,95 \% \mathrm{CI},-0.309$ :-0.039), poor baseline adherence (beta $=-0.145,95 \% \mathrm{CI},-0.214:-0.076$ ) and baseline weight (beta $=-0.004,95 \% \mathrm{CI},-0.006,-0.002$ ) were significant predictors of longitudinal CD4 change.

Table 3. Generalized linear mixed effect model for predictors of the changes in CD4 cell count over time for RVI patients on ART, 2013 to 2019.

\begin{tabular}{|c|c|c|c|c|c|}
\hline Variables & Category & beta & SE & P-Value & $95 \% \mathrm{CI}$ \\
\hline Intercept & & 5.777 & 0.084 & $<0.001$ & $(5.612,5.942)$ \\
\hline Time & & 0.306 & 0.010 & $<0.001$ & $(0.286,0.326)$ \\
\hline \multirow{2}{*}{ Sex } & Male & Ref. & Ref. & Ref. & Ref. \\
\hline & Female & 0.019 & 0.023 & 0.413 & $(-0.027,0.066)$ \\
\hline \multirow{7}{*}{ Occupational status } & Government employed & Ref. & Ref. & Ref. & Ref. \\
\hline & Non-government employed & 0.025 & 0.045 & 0.586 & $(-0.064,0.114)$ \\
\hline & Farmer & 0.027 & 0.050 & 0.598 & $(-0.073,0.126)$ \\
\hline & Student & -0.081 & 0.049 & 0.102 & $(-0.178,0.016)$ \\
\hline & Housewife & -0.069 & 0.047 & 0.134 & $(-0.161,0.022)$ \\
\hline & Merchant & 0.037 & 0.048 & 0.436 & $(-0.057,0.122)$ \\
\hline & Other* & 0.042 & 0.058 & 0.472 & $(-0.072,0.155)$ \\
\hline \multirow{2}{*}{ Place of residence } & Urban & Ref. & Ref. & Ref. & Ref. \\
\hline & Rural & 0.030 & 0.027 & 0.255 & $(-0.022,-0.083)$ \\
\hline \multirow{5}{*}{ Educational status } & Uneducated & Ref. & Ref. & Ref. & Ref. \\
\hline & Primary & 0.048 & 0.023 & 0.034 & $(0.004,0.092)$ \\
\hline & Secondary & 0.002 & 0.024 & 0.933 & $(-0.046,0.049)$ \\
\hline & Tertiary & 0.094 & 0.045 & 0.034 & $(0.007,0.182)$ \\
\hline & Diploma and above & 0.238 & 0.154 & 0.121 & $(-0.063,0.539)$ \\
\hline \multirow{4}{*}{ RVI stage } & Stage I & Ref. & Ref. & Ref. & Ref. \\
\hline & Stage II & -0.108 & 0.024 & $<0.001$ & $(-0.156,-0.061)$ \\
\hline & Stage III & -0.036 & 0.027 & 0.177 & $(-0.089,0.016)$ \\
\hline & Stage IV & 0.089 & 0.061 & 0.141 & $(-0.029,0.209)$ \\
\hline \multirow{3}{*}{ Functional } & Working & Ref. & Ref. & Ref. & Ref. \\
\hline & Ambulatory & -0.009 & 0.034 & 0.802 & $(-0.076,0.058)$ \\
\hline & Bedridden & -0.175 & 0.069 & 0.011 & $(-0.309,-0.039)$ \\
\hline \multirow{3}{*}{ Adherence } & Good & Ref. & Ref. & Ref. & Ref. \\
\hline & Fair & -0.034 & 0.054 & 0.520 & $(-0.140,0.071)$ \\
\hline & Poor & -0.145 & 0.035 & $<0.001$ & $(-0.214,-0.076)$ \\
\hline \multirow{2}{*}{ TB co-infection } & No & Ref. & Ref. & Ref. & Ref. \\
\hline & Yes & -0.046 & 0.025 & 0.072 & $(-0.096,0.004)$ \\
\hline Baseline weight & & -0.004 & 0.001 & $<0.001$ & $(-0.006,-0.002)$ \\
\hline Duration on ART & & 0.0001 & 0.001 & 0.911 & $(-0.0013,-0.0015)$ \\
\hline \multicolumn{6}{|l|}{ Variance component } \\
\hline SD of Intercept & & 0.754 & & & \\
\hline SD of Time & & 0.205 & & & \\
\hline
\end{tabular}

Other*: Daily worker, Drivers; Ref: Reference category; beta=regression coefficient.

\section{Discussion}

This longitudinal study examined the mean change in CD4 lymphocyte count and its predictors among HIV/AIDS patients on ART in West Hararghe zone, Ethiopia. The result of our analysis indicated time since start of ART, educational status, baseline WHO RVI stage, functional status, adherence, and weight are significantly associated with CD4 count change. Expected change in CD4 cell count for all patients at baseline is 5.78 , but shows substantial variation from one patient to another patient, with standard deviation of 0.75 .

Time since start of ART was strongly and significantly associated with longitudinal change in $\mathrm{CD} 4$ cell count. For a unit increase in time on ART the expected log count of CD4 cell increase 0.31 (beta $=0.306,95 \% \mathrm{CI}, 0.286: 0.326$ ). This 
finding is in agreement with previous study [10] and the possible reason is when patients took ART for long time adherence is more likely to have improved and this could result in increased CD4 count.

Patients with primary and tertiary level educational status are expected to have 0.05 and 0.03 higher log counts of CD4 cells as compared to uneducated ones (beta $=0.048,95 \% \mathrm{CI}$, 0.094: 0.092) and (beta $=0.034,95 \% \mathrm{CI}, 0.007$ : 0.082) respectively. This is consistent with findings from previous studies [11]. This could be explained by those patients who had access to education had better understanding on nutritious food. Therefore for educated ones proper nutrition increases the CD4 count among HIV infected adults [12].

For a unit increase in weight of patients the expected log count of CD4 cell decrease 0.004 (beta $=0.004,95 \% \mathrm{CI}$, $0.006,-0.002)$. This finding is in agreement with previous study in Ethiopia [13] and the possible reason for this is that excess weight is associated with elevated levels of inflammatory markers and complication which may have negative immune effects. Moreover, it can be explained by the fact that some lipophilic antiretroviral drugs are affected negatively by increased weight of RVI patients $[14,15]$.

Baseline functional status of patients are also significant predictor of change in CD4 count over time. Patients with bedridden functional status are expected to have 0.175 lower $\log$ counts of CD4 cells as compared patients with working functional status (beta $=-0.175,95 \% \mathrm{CI},-0.309$ : -0.039 ) and the result is supported by previous studies [13, 16]. This might be due to patients with bedridden baseline functional status might be affected susceptible to opportunistic infection and complications which in turn has negative effect on CD4 cell count.

According to our study patients with poor baseline adherence are expected to have 0.145 lower log counts of CD4 cells as compared to patients with good baseline adherence (beta $=0.145,95 \% \mathrm{CI},-0.214:-0.076$ ). The current study showed patients in WHO RVI stage II are expected to have 0.108 lower log counts of CD4 cells as compared to those in WHO RVI stage I (beta $=-0.108,95 \% \mathrm{CI},-0.156$, $0.061)$. The finding is in line with previous studies [13] and this is explained by RVI patients in higher stages are at more advanced stage of the disease, are prone to complication and this can reduce CD4 cell count.

The main limitation of the current study was since it was based on secondary data from patient records data couldn't be obtained for some important variables like nutritional status and substance use. Additionally, measurement of CD4 count was not done for patients as per schedule and not available for some patients.

\section{Conclusions}

Time since start of ART, primary and tertiary educational status contributed positively to the change in CD4 count whereas bedridden functional status, poor adherence, WHO RVI stage II and weight are negatively associated with longitudinal change in CD4 cell count. Close monitoring for bedridden patients and patients with poor baseline adherence is needed especially during the initiation of ART for immunological response. Further prospective cohort studies are needed to assess the effect of substance use and nutritional status on longitudinal change in CD4 cell count. We recommend health professionals to consistently measure CD4 count as per schedule for all patients.

\section{Acronyms and Abbreviations}

AIC: Akaike Information Criteria; AIDS: Acquired Immuno Deficiency Syndrome; ART: Anti-Retroviral Therapy; CI: Confidence Interval; HAART: Highly Active Anti-Retroviral Therapy;

HIV: Human Immunodeficiency Virus; RVI: Retroviral Infection; SD: Standard Deviation; SE: Standard Error; TB: Tuberculosis; WHO: World Health Organization.

\section{Competing Interests}

The authors declare that they have no competing interests.

\section{Funding}

The study was funded by the University of Gondar. The research was designed, conducted, analyzed and interpreted by the authors entirely independently of the funding source. The funder has no any role in the publication process.

\section{Availability of Data and Materials}

The datasets supporting the conclusions of this article are available upon request to the corresponding author.

\section{Consent for Publication}

Not applicable.

\section{Ethical Approval}

Ethical clearance for selected Hospitals was obtained from the Institutional Review Board of the University of Gondar. Confidentiality during all phases of research activities was kept. No personal identifiers like name and private information was collected.

\section{Author Contributions}

ABW conceptualized the study, collects the data, analyzed the data and drafted the manuscript. BST, SAK and GAD also participated in designed the study, analyzed the data and critically revised the manuscript. All authors read and approved the final manuscript.

\section{Acknowledgements}

We thank University of Gondar for funding and giving 
ethical clearance to conduct this research. Our special thanks also goes to Hawulte Nigussie, Feven Zenebe and Hiwot for their support during data collection period.

\section{References}

[1] WHO, AIDS statistics 2018 fact sheet. 2019; 31. 2019.

[2] Kharsany, A. B. and Q. A. J. T. o. A. j. Karim, HIV infection and AIDS in sub-Saharan Africa: current status, challenges and opportunities. 2016. 10: p. 34.

[3] UNAIDS, W. and W. J. L. G. U. Cameroon, WHO, Epidemiological Fact Sheet on HIV/AIDS and Sexually Transmitted Infections, 2002 update. 2002.

[4] Osler, M., et al., The continuing burden of advanced HIV disease over 10 years of increasing antiretroviral therapy coverage in South Africa. 2018. 66 (suppl_2): p. S118-S125.

[5] McCune, J. M. J. N., The dynamics of CD4+ T-cell depletion in HIV disease. 2001.410 (6831): p. 974-979.

[6] Low-Beer, S., et al., Adherence to triple therapy and viral load response. 2000. 23 (4): p. 360-361.

[7] Thompson, M. A., et al., Guidelines for improving entry into and retention in care and antiretroviral adherence for persons with HIV: evidence-based recommendations from an International Association of Physicians in AIDS Care panel. 2012. 156 (11): p. 817-833.

[8] WHO, Guidelines on post-exposure prophylaxis for HIV and the use of co-trimoxazole prophylaxis for HIV-related infections among adults, adolescents and children: recommendations for a public health approach: December 2014 supplement to the 2013 consolidated guidelines on the use of antiretroviral drugs for treating and preventing HIV infection, in Guidelines on post-exposure prophylaxis for HIV and the use of co-trimoxazole prophylaxis for HIV-related infections among adults, adolescents and children: recommendations for a public health approach: December 2014 supplement to the 2013 consolidated guidelines on the use of antiretroviral drugs for treating and preventing HIV infection. 2014.

[9] IeDEA, T. J. J. o. a. i. d. s., Immunodeficiency at the start of combination antiretroviral therapy in low-, middle-and highincome countries. 2014. 65 (1): p. e8.

[10] Smith, C. J., et al., Factors influencing increases in CD4 cell counts of HIV-positive persons receiving long-term highly active antiretroviral therapy. 2004. 190 (10): p. 1860-1868.

[11] Seyoum, A., Z. J. A. r. Temesgen, and therapy, Joint longitudinal data analysis in detecting determinants of CD4 cell count change and adherence to highly active antiretroviral therapy at Felege Hiwot Teaching and Specialized Hospital, North-west Ethiopia (Amhara Region). 2017. 14 (1): p. 14.

[12] Maurya, S., et al., The level of education affects CD4 cell count and wellness among HIV infected adult between age group 18 to 60 years. 2016. 45: p. 264.

[13] Reda, A. A., et al., Predictors of change in CD4 lymphocyte count and weight among HIV infected patients on antiretroviral treatment in Ethiopia: a retrospective longitudinal study. 2013.8 (4).

[14] Tanaka, S. I., et al., T lymphopaenia in relation to body mass index and TNF- $\alpha$ in human obesity: adequate weight reduction can be corrective. 2001.54 (3): p. 347-354.

[15] Lord, G. M., et al., Leptin inhibits the anti-CD3-driven proliferation of peripheral blood $\mathrm{T}$ cells but enhances the production of proinflammatory cytokines. 2002. 72 (2): p. 330-338.

[16] Gezie, L. D. J. B. r. n., Predictors of CD4 count over time among HIV patients initiated ART in Felege Hiwot Referral Hospital, northwest Ethiopia: multilevel analysis. 2016. 9 (1): p. 377. 